NBER WORKING PAPER SERIES

\title{
WHY HAVE HEALTH EXPENDITURES AS A SHARE OF GDP RISEN SO MUCH?
}

\author{
Charles I. Jones \\ Working Paper 9325 \\ http://www.nber.org/papers/w9325 \\ NATIONAL BUREAU OF ECONOMIC RESEARCH \\ 1050 Massachusetts Avenue \\ Cambridge, MA 02138 \\ November 2002
}

I would like to thank David Cutler, Ryan Edwards, Victor Fuchs, Ted Keeler, Ron Lee, Mark McClellan, Patrizio Pagano, Jim Poterba, David Romer, Emmanuel Saez, Robert Topel, Alwyn Young, and participants of seminars at an NBER EFG meeting, the San Francisco Fed, U.B.C., U.C. Berkeley, U.C. Davis, U.C. Riverside, and U.S.C. for very helpful comments. Meredith Beechey and Jesse Czelusta provided excellent research assistance. Financial support from a John M. Olin Foundation Faculty Fellowship and an Alfred P. Sloan Research Fellowship is gratefully acknowledged. I would also like to thank the Federal Reserve Bank of San Francisco for hosting me as a visiting scholar during portions of this research. The views expressed herein are those of the author and not necessarily those of the National Bureau of Economic Research.

(C) 2002 by Charles I. Jones. All rights reserved. Short sections of text, not to exceed two paragraphs, may be quoted without explicit permission provided that full credit, including $(\mathcal{C}$ notice, is given to the source. 
Why Have Health Expenditures as a Share of GDP Risen So Much?

Charles I. Jones

NBER Working Paper No. 9325

November 2002

JEL No. I1, O40

\begin{abstract}
Aggregate health expenditures as a share of GDP have risen in the United States from about 5 percent in 1960 to nearly 14 percent in recent years. Why? This paper explores a simple explanation based on technological progress. Medical advances allow diseases to be cured today, at a cost, that could not be cured at any price in the past. When this technological progress is combined with a Medicare- like transfer program to pay the health expenses of the elderly, the model is able to reproduce the basic facts of recent U.S. experience, including the large increase in the health expenditure share, a rise in life expectancy, and an increase in the size of health-related transfer payments as a share of GDP.
\end{abstract}

\author{
Charles I. Jones \\ Department of Economics \\ University of California, Berkeley \\ Berkeley, CA 94720-3880 \\ and NBER \\ chad@econ.berkeley.edu
}


FIGURE 1. OECD Health Expenditures as a Share of GDP

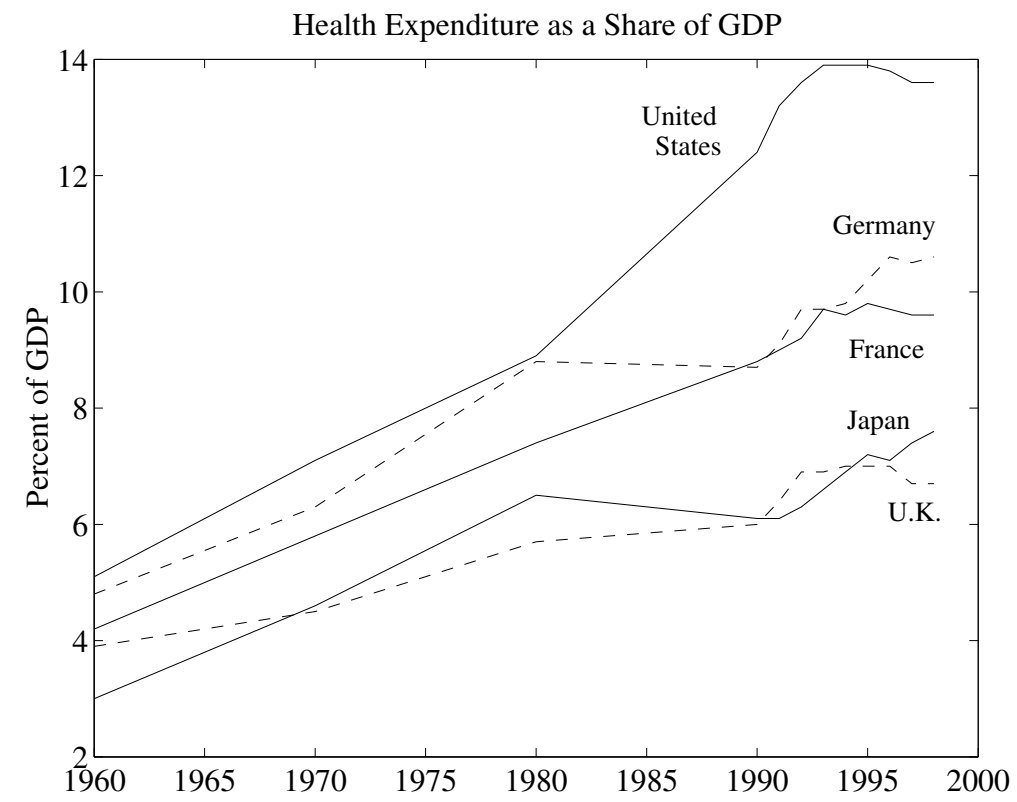

Source: OECD (2000), Table 16.

\section{INTRODUCTION}

In 1960, aggregate health expenditures in the United States were 5.1 percent of GDP; by 1997, the health expenditure share stood at 13.6 percent. Why have health expenditures as a share of GDP risen so dramatically?

One conventional explanation holds that U.S. government policy is responsible. Changes in transfer programs such as Medicare and Medicaid have possibly led to the increased spending on health. One problem with this explanation, however, is that similar increases in health expenditures as a share of GDP have been observed throughout the OECD, as shown in Figures 1 and 2. It is true, as is often noted, that the United States spends a larger fraction of its income on health than other countries, but the recent tendency for this expenditure ratio to increase seems to be a widespread 
FIGURE 2. OECD Health Share vs. Life Expectancy, 1960 and 1997

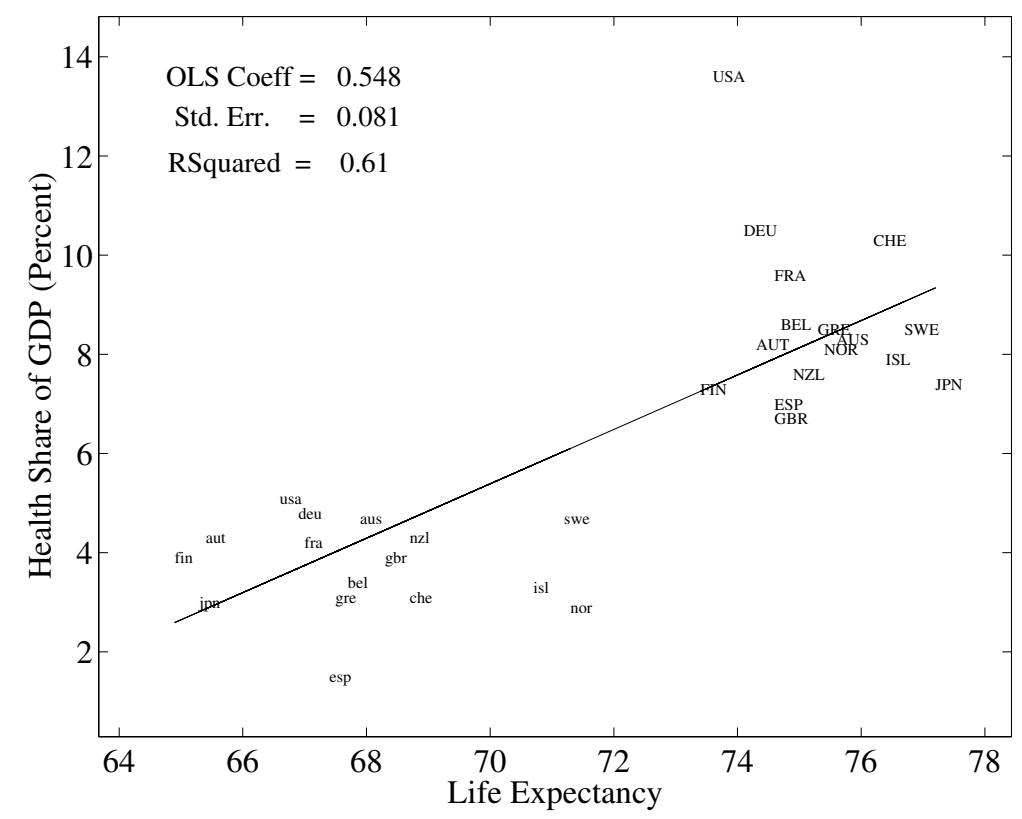

Notes: Observations for 1960 are plotted by country code with lowercase letters; uppercase letters correspond to observations for 1997. Data from OECD (2000), Tables 7 and 16. Life Expectancy is for males only.

phenomenon. The typical change across the OECD is an increase in the health expenditure share from about 4 percent of GDP to about 8 percent.

Another possible explanation is the cost disease story of Baumol (1967). According to this explanation, health care, like other services, uses labor intensively and exhibits relatively low productivity growth. This leads to a rising relative price of health care and, depending on preferences, to a rising share of expenditures. Superficially, this argument appears somewhat plausible, as measured labor productivity growth in the health sector was negative between 1987 and 1997 and the medical care component of the CPI grew faster than the overall CPI (Triplett and Bosworth (2000)). On 
the other hand, the general cost disease explanation for health care rings hollow in the face of rapid technological advances such as the discovery of new drugs, diagnostic equipment, and medical procedures that appear to be occurring throughout the health sector. Moreover, it is clear that measurement problems in this sector are severe, and attempts to correct these measurement problems support the casual observation that medical care is a sector with rapid technological change. As just one example, Cutler, McClellan, Newhouse and Remler (1998) report that between 1983 and 1994, the real quality-adjusted price of heart attack treatments actually declined at an annual rate of 1.1 percent. $^{1}$

Newhouse (1992) provides a nice survey of possible explanations for the rise in health expenditures as a share of GDP. In addition to changes in U.S. policy and the Baumol cost-disease story, Newhouse considers and rejects explanations driven by the increase in health insurance, increased income and health as a luxury good, and supplier-induced demand for medical care. For example the income effects explanation is problematic in large part because estimates of the income elasticity of demand for medical care in the United States, holding insurance constant, are only about 0.2 to 0.4 .

After reviewing in detail these possible explanations, Newhouse finds that he is left with a large residual: these explanations account for only a minority of the increase in health expenditures, certainly less than half and perhaps less than a quarter. Instead, Newhouse argues that "I believe the bulk of the residual increase is attributable to technological change, or what might loosely be called the march of science and the increased capabilities of medicine" (p. 11).

\footnotetext{
${ }^{1}$ Triplett (forthcoming) shows how such corrections of price indexes can impact measured productivity. As an exercise, he applies a price index that shows zero inflation in medical care to the entire health sector. With this change, multifactor productivity in health services increases at a rate of 5.6 percent per year between 1987 and 1997, rather than declining at an annual rate of 2.6 percent.
} 
This paper constructs a simple model to examine the effects of the "march of science" on health expenditures. Technological advances allow diseases and other health problems that could not be cured at any price in the past to be treated effectively today. Consider the development of MRIs, arthroscopic medical procedures, antibiotics, angioplasty, and drugs that treat depression. Before these and other technologies were discovered, health expenditure shares were low by default. The gradual discovery of new treatments over time directly increases the expenditure share by permitting the desired health expenditures to occur.

Of course, a countervailing force is that technological progress continually reduces the cost of any given treatment, as in the heart attack example given above. MRIs and heart attack treatments — once adjusted for quality — are much cheaper today than a decade ago. How these two forms of technological progress ultimately influence aggregate health expenditures is something this paper will explore.

Another important component of the model is a Medicare-like transfer program. As modeled, this program features an endogenous tax rate that adjusts to ensure that the elderly live as long as is technologically feasible. This transfer system and technological progress turn out to be a powerful combination. Unchecked, this combination produces a rapidly growing health share that can eventually consume an arbitrarily large fraction of GDP. A critical parameter of the model then turns out to be the maximum transfer rate that society is willing to tolerate.

The paper is organized in the following way. Section 2 develops the basic model, while Section 3 adds the transfer program. Section 4 shows that the model, for plausible parameter values, is consistent with the Newhouse view that the march of science accounts for the majority of the increase in the aggregate spending share. Section 5 provides a fully worked-out simulation of the model for a particular parameterization. Section 6 presents some 
additional evidence on the validity of the model, and Section 7 offers some concluding remarks.

\section{BASIC ACCOUNTING IN A SIMPLE MODEL}

In this section we present a very stylized model meant to capture some of the facts related to health expenditures. The model neglects many aspects of the reality of medical care in order to keep the model as simple as possible and to focus on the role of technological progress.

\subsection{Microfoundations}

Suppose there are a range of health problems that can afflict a person. These problems are indexed by $x \in[0, \infty)$ and are ordered so that health problems with a higher $x$ are scientifically more difficult to treat. At any point in time, successful treatments have been discovered only for health problems in the range $[0, \bar{x}(t)]$.

Let $\ell(x, t)$ denote life expectancy for someone successfully treated for condition $x$ at time $t$. In modern actuarial tables, life expectancy has a statistical meaning, different from the number of years a typical person might expect to live, which depends on future technological progress. To avoid making assumptions about the future, life expectancy is calculated as the expected length of life for a person born today and forever facing the mortality rates that prevail in the cross-section of ages today. With one change, we will give a similar interpretation to $\ell$. The change is that $\ell$ measures the expected length of life assuming all technologically-available treatments are utilized. In fact, as we will see, some technologies may be so expensive that they will not be utilized and people will die who, at some cost, could live longer. We assume that $\ell(x, t)$ is given by

$$
\ell(x, t)=\bar{x}(t)-x .
$$


Life expectancy is higher for less severe conditions, but falls to zero for someone who contracts the most severe health problem that can be treated, $\bar{x}(t)$. In the absence of a treatment for any health problem, a person dies.

Once a treatment is discovered, the cost of treating condition $x$ is

$$
\bar{h}(x, t)=\bar{h}_{0} e^{\beta x-\gamma(t-\delta(x))},
$$

where $\delta(x)$ denotes the date at which a successful treatment for condition $x$ is first discovered. The cost of treating a disease when the treatment is first discovered increases at rate $\beta$ with the severity of the disease. However, after the initial treatment is discovered, subsequent technological progress continually reduces the cost of treating the disease at rate $\gamma$. In the model, then, technological progress affects health expenditures in two different ways. The march of science leads to the discovery of costly treatments for health problems that could previously not be cured, but it also reduces the cost of those treatments over time.

We model both kinds of technological progress as exogenous and assume that the date at which the first successful treatment for condition $x$ is discovered is given by

$$
\delta(x)=\alpha\left(x-\bar{x}_{0}\right)
$$

where $\bar{x}_{0}$ represents the frontier of medical knowledge at time $t=0$. Every $\alpha$ years, the march of science shifts this frontier out by one unit.

\subsection{Some useful implications}

We now derive the distribution of health expenditures across people as a function of life expectancy, and we then derive the time series process for economy-wide life expectancy. These derivations are particularly useful when it comes time to match the model up with data. 
Some straightforward algebra shows that ${ }^{2}$

$$
h(\ell, t) \equiv \bar{h}(x(\ell), t)=h_{0} e^{\mu t-\theta \ell},
$$

where

$$
\mu \equiv \beta / \alpha
$$

and

$$
\theta \equiv \beta+\alpha \mu=\alpha(\mu+\gamma)
$$

Health expenditures are highest for people near death and decline exponentially across people at rate $\theta$ as life expectancy rises. A person with a lower life expectancy has higher health expenditures for two reasons, as can be seen in equation (6). First, the person suffers from more serious medical problems, which increase the cost of treatment at rate $\mu$. Second, the initial treatments for these more serious medical problems have been discovered more recently, meaning that the cost-reducing technological progress associated with $\gamma$ has had less time to reduce the cost of treatment. ${ }^{3}$

Equation (4) also describes how this distribution of health expenditures shifts over time. At a given level of life expectancy, health expenditures rise over time at rate $\mu \equiv \beta / \alpha$. As the presence of the parameter $\beta$ in this expression makes clear, this increase reflects the fact that people at a given level of life expectancy suffer from more severe - and therefore more costly - medical problems over time. The sickest person in the economy in 2002 is much more costly to treat than the sickest person in 1950 because technological progress has significantly shifted out the frontier medical condition that can be treated.

\footnotetext{
${ }^{2}$ First, notice that $\bar{x}(t)=\bar{x}_{0}+t / \alpha$, so that $x=\bar{x}_{0}+t / \alpha-\ell$. Substituting this relation together with the equation describing $\delta(x)$ gives the desired result, where $h_{0} \equiv \bar{h}_{0} e^{\beta \bar{x}_{0}}$.

${ }^{3}$ The parameter $\alpha$ in this equation plays the role of an exchange rate, converting between parameters describing growth over time $(\mu$ and $\gamma$ ) and a parameter that describes a growth rate across ages $(\theta)$.
} 
A more straightforward implication of the model relates to economywide life expectancy. The highest life expectancy in the population is given by $\ell(0, t)=\bar{x}(t)=\bar{x}_{0}+t / \alpha$, where the last equality comes from setting $t=\delta(\bar{x}(t))$ in equation (3). This corresponds nicely to what demographers calculate as life expectancy at birth, and it will be useful to give this quantity its own variable name, $\bar{a}(t)$ :

$$
\bar{a}(t) \equiv \ell(0, t)=\bar{x}_{0}+\frac{t}{\alpha} .
$$

Because of technological progress, economy-wide life expectancy rises by 1 year every $\alpha$ years. White (2002) shows that this linear formulation provides an excellent fit of the data in high-income countries in the second half of the twentieth century with $\alpha$ approximately equal to 5 .

\subsection{Remaining setup}

The remaining setup of the model involves the distribution of the population, timing considerations, and a description of income. For the sake of simplicity, we assume that the population is uniformly distributed across life expectancy at each point in time, with $\ell \in\left[\ell^{*}(t), \bar{a}(t)\right] .{ }^{4}$ The endogenous variable $\ell^{*}(t)$ will be equal to zero if all available technologies are utilized. It will be greater than zero to the extent that some technologies are not used. For example, suppose that $\ell^{*}=2$ at some time. In this case, people die when they, if all treatments were used, could live for two additional years. This might be an outcome if those additional treatments are extraordinarily expensive. We will define

$$
a^{*}(t) \equiv \bar{a}(t)-\ell^{*}(t)
$$

This can be thought of as actual life expectancy at birth in the population at time $t$, and, since the population is uniformly distributed across $\ell$, it denotes

\footnotetext{
${ }^{4}$ From equation (1), we are essentially assuming a uniform distribution across health conditions.
} 
the size (measure) of the population at time $t$. Note that the assumption that the population remains uniformly distributed across $\ell$ while life expectancy is growing means that there is population growth.

The timing of the model works as follows. Time is discrete. At the beginning of a period, people undertake their health expenditures, and then live for that period. People whose life expectancies in period $t$ are in the range $\left[\ell^{*}(t), \ell^{*}(t)+1\right)$ die at the end of that period.

Finally, let $Y(t)$ denote aggregate income at time $t$ so that $y(t) \equiv$ $Y(t) / a^{*}(t)$ is per capita income. We assume $y(t)=y_{0} e^{g t}$ so that per capita income grows exogenously at the constant rate $g$. At this point, we do not need to make any assumptions about the distribution of this income across people.

\subsection{Analysis}

With this setup, we are ready to solve for aggregate health expenditure as a share of GDP. First notice that total health expenditure, $H(t)$, is given by

$$
H(t) \equiv \int_{\ell^{*}(t)}^{\bar{a}(t)} h(\ell, t) d \ell
$$

Dividing by GDP and recalling that $Y(t)=a^{*}(t) y(t)$ yields

$$
\frac{H(t)}{Y(t)}=\frac{1}{a^{*}(t)} \int_{\ell^{*}(t)}^{\bar{a}(t)} \frac{h(\ell, t)}{y(t)} d \ell .
$$

That is, the aggregate expenditure share is the average of the individual expenditure shares. ${ }^{5}$

\footnotetext{
${ }^{5}$ Notice that we are abusing the language a bit with this statement. Because we've made no assumptions about the distribution of income, the correct statement would be that the aggregate share is a weighted average of the individual expenditure shares, where the weights correspond to income shares. That is, we could multiply and divide the term inside the integral by $y(\ell, t)$. Rather than proceed in this way, it is convenient to leave the expression as $h(\ell, t) / y(t)$. We will speak of this as the health expenditure share by someone with life expectancy $\ell$, but the income-weight caveat should be kept in mind.
} 
Using equation (4), it is straightforward to show that

$$
\begin{aligned}
\frac{H(t)}{Y(t)} & =\frac{1}{\theta a^{*}(t)}\left(\frac{h\left(\ell^{*}, t\right)}{y(t)}-\frac{h(\bar{a}(t), t)}{y(t)}\right) \\
& =\frac{1}{\theta a^{*}(t)} \frac{h\left(\ell^{*}, t\right)}{y(t)}\left(1-e^{-\theta a^{*}(t)}\right) .
\end{aligned}
$$

The first line of this expression exploits a simple fact that the integral of an exponential process is proportional to the difference between its value at the two endpoints. ${ }^{6}$ The second line takes advantage of the fact that this difference is proportional to the expenditure share by the person closest to death.

Finally, as long as $\theta a^{*}(t)$ is relatively large, a very good approximation is given by

$$
\frac{H(t)}{Y(t)} \approx \frac{1}{\theta a^{*}(t)} \frac{h\left(\ell^{*}, t\right)}{y(t)} .
$$

There are several things to note about this result. First, the aggregate health expenditure share is proportional to the largest quantity of health expenditures in the population divided by per capita GDP. Let us call this term, $h\left(\ell^{*}, t\right) / y(t)$, the health expenditure share at the end of life, because it reflects the health expenditures of individuals with the minimal level of life expectancy. It is slightly misleading to call it an expenditure share because it is per capita income in the denominator rather than any individual's income. Still, it is convenient to have a short phrase to describe this quantity.

Second, the factor of proportionality depends inversely on life expectancy, $a^{*}(t)$. Holding constant the expenditure share of people near death, an increase in economy-wide life expectancy reduces the aggregate expenditure

\footnotetext{
${ }^{6} \mathrm{~A}$ more intuitive way to view this mathematical fact is to consider the average of an exponential process rather than the sum. If $x(t)=x_{0} e^{g t}$, then $\bar{x}(t) \equiv \frac{1}{T} \int_{0}^{T} x(t) d t$ is equal to $1 / g T \times(x(T)-x(0))$. Rearranging, we see that $g=1 / T \times(x(T)-x(0)) / \bar{x}$. The exponential growth rate is equal to the percentage change in the process, where the change is taken relative to, not the starting or ending value, but to the exponential average itself.
} 
share. We will call this the dilution effect. Recall that the aggregate spending share is the average of the individual spending shares. A higher level of life expectancy, with the highest expenditure share held constant, essentially means a larger measure of low-cost healthy people over which we are averaging. At any point in time, there are always five years worth of people who are five years away from dying. This is true whether economy-wide life expectancy is 60 years or 80 years, but in the latter case, these five years worth of people constitute a smaller fraction of the population.

Third, because of this dilution effect, growth in the aggregate expenditure share requires the expenditure share at the end of life to grow faster than life expectancy. Using equation (4), we can replace this expenditure share with the appropriate exponential terms to get

$$
\frac{H(t)}{Y(t)} \approx \frac{1}{\theta a^{*}(t)} \frac{h_{0}}{y_{0}} e^{(\mu-g) t} e^{-\theta \ell^{*}}
$$

A necessary condition for $H / Y$ to grow is that $\mu>g$, i.e. that health expenditures at a given level of life expectancy grow faster than per capita GDP.

Finally, we can use the approximation in equation (14) to get a rough sense of the numbers involved. As discussed in the introduction, between 1960 and 1997, the U.S. health expenditure share rose from 5.1 percent to 13.6 percent, that is by a factor of $13.6 / 5.1=2.7$. Similarly, life expectancy over this period rose from 66.6 years to 73.9 year, or by a factor of $73.9 / 66.6=1.11$, according to the data in Figure 2. To match these numbers, the expenditure share at the end of life must rise by a factor of $2.7 \times 1.11=3.0$. Assuming $\ell^{*}$ is constant and $g=.018$, this requires a value of $\mu=.047$. That is, health expenditures by people shortly before they die must have been rising at a rate of 4.7 percent per year.

By itself, this result is not surprising - it is simply a matter of accounting. If $H / Y$ has been rising and if health expenditures are distributed exponen- 
tially across the population, then the health expenditure share at the end of life must have been rising, and we have simply calculated the rate at which this rise must occur.

The careful reader may now be bothered by something: if the expenditure share of decedents grows without bound, what prevents $H / Y$ from growing beyond 100 percent? The answer, of course, must be that the expenditure share at the end of life cannot grow without bound: at some point, individual health expenditures relative to per capita income must level off. Since we have not discussed the economic forces governing an individual's choice of health expenditure, this result has been suppressed; it will be explored in detail in the next section.

Still, we can pause to see the basic implication of this bound. Suppose that $h\left(\ell^{*}, t\right) / y(t)$ reaches an upper bound and stops growing. From equation (13), the dilution effect will then dominate and $H / Y$ declines, eventually asymptoting to zero. Recall from equation (10) that the aggregate expenditure share is simply the average of the individual expenditure shares. With the top expenditure share bounded, this average is continually diluted by the fact that a rising life expectancy introduces an increasing number of low-cost healthy individuals.

\section{PAY-AS-YOU-GO HEALTH CARE FINANCING}

To this point, we have suppressed any budget constraint considerations in the determination of health expenditures. This neglect is now remedied. The individual expenditure decision is impacted significantly by public and private health insurance and by life cycle considerations. Rather than analyze this complicated choice, we make a number of special assumptions about the economics of individual decision-making. The goal is to build a model that is simple to analyze yet sophisticated enough to produce interesting results. 
At some point, individuals approach the end of life and face large health expenses. We assume that these expenses are financed in the following way. First, individuals near the end of life have income and wealth that allows them to spend up to some multiple, $\sigma$, of disposable per capita income on health care. For simplicity, the level of this resource constraint is assumed to be the same across people. The parameter $\sigma$ is a reduced-form way to capture several competing concerns. On the one hand, individuals may have access to health insurance, discussed below, so that they only need to pay some fraction of their health expenses, leaving more resources for consumption and bequests. This suggests that $\sigma$ might be low. On the other hand, individuals know that they will face large health expenses near the end of life and may save for this purpose. This would make $\sigma$ high. Clearly, $\sigma$ could be greater or less than one, and in a richer model would not be invariant to the policy regime: changes in the health care system would change $\sigma$. Modeling these sophisticated effects is important. However, it is also complicated, and the results that one obtains are likely to be sensitive to the exact nature of private and public health insurance and to behavioral assumptions. We leave this valuable research to future work and instead focus on the simpler case where $\sigma$ is a reduced-form parameter, with these caveats kept in mind.

In addition to their own resources, individuals in this model have access to a basic health insurance system. We will think of this as public health insurance, but there is no reason in the model why it could not be run privately. The insurance scheme is financed by a flat income tax (premium) at rate $\tau(t)$. It pays out benefits, $v(\ell, t)$, that depend on health status.

Because of rapid technological progress, the cost of keeping people alive in the last year of life rises more rapidly than per capita income $(\mu>g)$. At some point, these costs outstrip an individual's ability to pay for treatments, so that even though the technology exists to prolong life, the cost is greater 
than the individual can afford. Call the date at which this occurs $t^{*}$, and notice that this date solves the following equation

$$
h\left(0, t^{*}\right)=\sigma y(t)
$$

Prior to date $t^{*}$, individuals can pay their own health expenses, and we will assume the insurance scheme does not operate, so that $\tau(t)=0$ for $t<t^{*}$. Up until this date, aggregate health expenses are determined by equation (13) with $\ell^{*}(t)=0$. That is, people live as long as is technologically possible.

After date $t^{*}$, this changes. The technological costs of keeping individuals alive as long as possible may exceed their resources. In the absence of a transfer scheme, such people would die immediately.

Transfers $v(\ell, t)$ are paid to these people in exactly the quantity needed to keep them alive until the budget is exhausted. That is

$$
v(\ell, t)=h(\ell, t)-\sigma(1-\tau(t)) y(t), \quad \ell \in\left[\ell^{*}(t), \bar{\ell}(t)\right],
$$

where $\bar{\ell}(t)$ denotes the life expectancy of the marginal person requiring a transfer, given implicitly by

$$
h(\bar{\ell}(t), t)=\sigma(1-\tau(t)) y(t) .
$$

Finally, we assume that the transfer program operates with a balanced budget:

$$
\tau(t) a^{*}(t) y(t)=\int_{\ell^{*}(t)}^{\bar{\ell}(t)} h(\ell, t)-h(\bar{\ell}(t), t) d \ell .
$$

We make the following additional assumptions about the insurance institution. After date $t^{*}$, transfer payments are used to keep people alive as long as is technologically feasible. That is, equation (18) can be thought of as determining the insurance premium $\tau(t)$ so that $\ell^{*}(t)=0$. We also 
assume that this premium/tax rate is capped at $\bar{\tau}<1$. In general, there will be a third date $t^{* *}$ at which time this bound on the tax rate is binding. In this regime, equation (18) can be thought of as determining $\ell^{*}(t)$ so that the budget constraint is satisfied with $\tau(t)=\bar{\tau}$. That is, life expectancy is rationed by the willingness of society to pay the large health expenses of those near death.

We pause at this point to highlight the key endogenous variables and the key equations that determine them. These variables are $h(\ell, t), \bar{a}(t), a^{*}(t)$, $\ell^{*}(t), \bar{\ell}(t), H(t) / Y(t)$, and $\tau(t)$. The equations that determine these endogenous values are (4), (7), (8), (17), (18), (13), and the conditions described above that set $\tau(t)=0$ when $t \leq t^{*}, \ell^{*}(t)=0$ for $t \leq t^{* *}$, and $\tau(t)=\bar{\tau}$ when $t>t^{* *}$. Notice that $t^{*}$ is determined by equation (15) and $t^{* *}$ is determined as the date at which $\tau\left(t^{* *}\right)=\bar{\tau}$ and $\ell^{*}\left(t^{* *}\right)=0$ in equation (18).

\section{SIMULATING THE MODEL, PART I}

The survey by Newhouse (1992) suggested that the "march of science" was responsible for at least 50 to 75 percent of the rise in U.S. health expenditures. Now that we have a model in which technological progress can lead to a rising health share, the question is whether or not the magnitude of the increase is quantitatively significant for plausible parameterizations.

Values for some parameters are relatively easy to infer from various stylized facts. For the underlying growth rate of per capita income, $g$, we take a value of 1.8 percent, corresponding roughly to the growth rate of GDP per capita in the United States in the postwar period. The parameter $\alpha$ measures the number of years it takes technological progress to raise life expectancy by a year. In the United States, male life expectancy increased from 66.6 years in 1960 to 73.9 years in 1997, suggesting a value of $\alpha$ of 
$(1997-1960) /(73.9-66.6)=5.07$, consistent with the estimates for advanced countries since 1955 in White (2002).

Picking a value for $\theta$ gives us the opportunity to examine two different pieces of data. Recall that $\theta$ captures the rate at which health expenditures decline with life expectancy in a cross-section of the population. Miller (2001) reports average annual Medicare expenditures per enrollee for 129,166 randomly-chosen people who died in 1989 or 1990, using data from Lubitz, Beebe and Baker (1995). Data from the Continuous Medicare History Sample tracks their Medicare expenditures back to 1974, allowing Miller to look at Medicare expenditures versus years before death. ${ }^{7}$ These numbers are plotted in Figure 3. While the model assumes this relation to be log-linear, the data suggest that it is not. Rather, a log-linear spline seems to fit the data better. Medicare expenditures rise at a rate of 9.4 percent per year for people 3 to 10 years away from death. This rate then accelerates sharply to 45 percent per year in the last two years before death. This suggests a value of $\theta$ in the model that is some average of 0.094 and $0.45 .^{8}$

Fortunately, $\theta$ can also be calculated using a second piece of data. It is straightforward to show (see the Appendix) that the fraction of total health expenditures accounted for by people in the last year of life is approximately equal to $1-e^{-\theta} \approx \theta$. Intuitively, this occurs because the distribution of

\footnotetext{
${ }^{7}$ Since the data correspond to Medicare expenditures over time for people who died in a single year, one might worry that Figure 3 reveals $\mu+\theta$ rather than just $\theta$. Footnote 3 in Miller (2001) documents that the expenditures are placed in 1990 dollars by multiplying by a factor corresponding to per capita Medicare expenditures in the two years. This essentially undoes the $\mu$ effect. In terms of the model, it introduces an extra term corresponding to the proportion by which life expectancy changed between the two years, but this factor is extremely small relative to the $\theta$ effects, so I have chosen to report the original Miller data rather than making the correction.

${ }^{8}$ The model could be augmented to capture this non-exponential pattern by adding a term $\gamma y(t)$ to the basic spending relation in equation (4). This term incorporates health expenditures that are largely independent of life expectancy, such as healthy pregnancy and childbirth expenses, vaccinations, athletic injuries, etc.
} 
FIGURE 3. Average Annual Medicare Expenditures per Enrollee

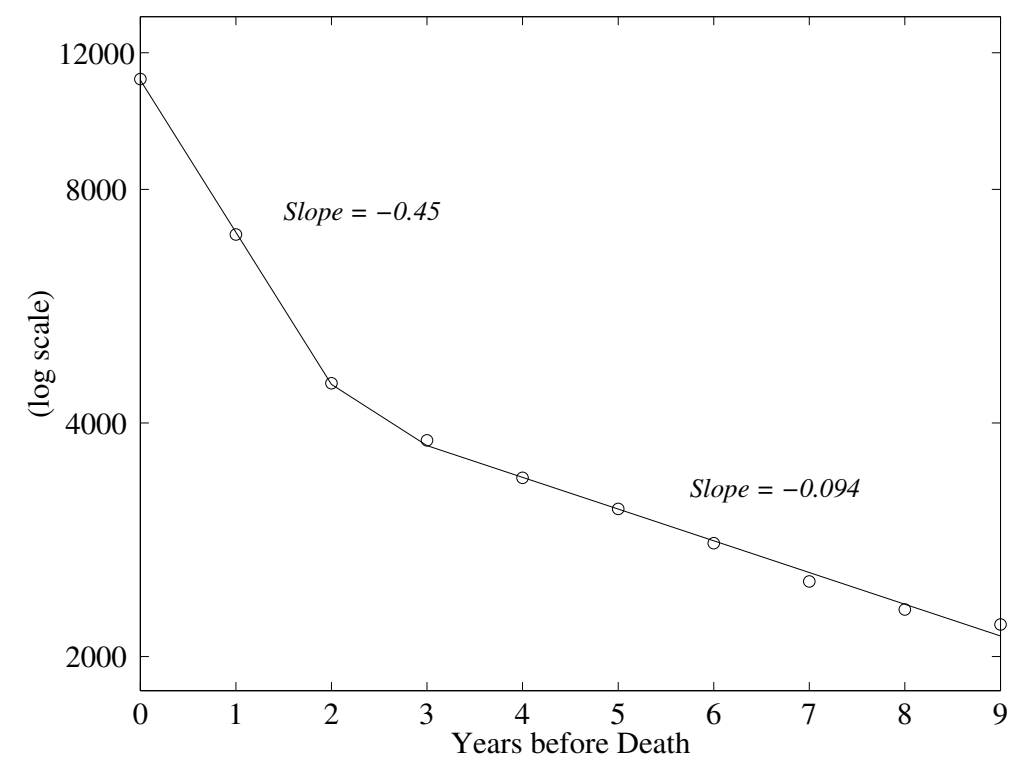

Circles represent average expenditures for all enrollees over age 65, taken from Miller (2001), Table 1.

health expenditures across people is exponential with parameter $\theta$, and this entire distribution shifts in the same way over time. Lubitz and Riley (1993) document that the share of Medicare payments on behalf of people in the last year of life is relatively stable, fluctuating between 27.2 and 30.6 percent over the period 1976 to 1988 . This is useful in two ways. First, Lubitz and Riley emphasize that their result is somewhat surprising: many people had suspected that the large rise in Medicare expenditures was disproportionately associated with people in the last year of life. They show that this is not the case, and the model also predicts that this fraction should be stable. Second, we can use these numbers to help calibrate $\theta$. Presumably the fraction of total health expenditures occuring in the last year 
of life is smaller than the fraction for Medicare. ${ }^{9}$ Based on this fraction and on the Miller evidence discussed above, we choose $\theta=0.2877$ so that $1-e^{-\theta}=0.25$ as a benchmark value, i.e. one quarter of total health expenditures are assumed to go for people in the last year of life.

The final parameter - and in fact really the crucial parameter of the model - is $\mu$. As was apparent in the calculations at the end of Section 2, with a sufficiently large value of $\mu$ the model will have no trouble explaining the nearly 3 -fold increase in the U.S. health share. To make a more informed judgment as to how much of the increase in $H / Y$ the model can explain, one would ideally like to obtain the value of the parameter $\mu$ without reference to the magnitude of the increase in the aggregate health expenditure share. This is what we do now.

For this purpose, the key insight comes from equation (6), which we reproduce here:

$$
\theta=\alpha(\mu+\gamma)
$$

Recall that $\theta$ measures the rate at which health expenditures change in the cross section with a change in life expectancy. Health expenditures are higher for a person with lower life expectancy for two reasons. First, the health problems possessed by this person are more severe, raising costs at rate $\mu$. Second, basic treatments for these more severe problems have been discovered more recently, meaning that the cost-reducing technological progress occuring at rate $\gamma$ has had less time to operate.

If we knew the value of $\gamma$, equation (19) could be used to back out an implied value of $\mu$. Fortunately, estimates of $\gamma$ are now available from recent work in health economics. This parameter corresponds empirically to the annual rate at which the quality-adjusted cost of treating a specific medical

\footnotetext{
${ }^{9}$ This is not entirely clear, however, since many nursing home expenses are not covered by Medicare.
} 
condition declines over time. As discussed in the introduction, Cutler et al. (1998) find that the real quality-adjusted price for treating heart attacks declines at a rate of 1.1 percent per year between 1983 and 1994. Other authors report similar findings. For example, Shapiro, Shapiro and Wilcox (1999) examine the treatment price for cataracts between 1969 and 1994. While a CPI-like price index increased at an annual rate of 9.2 percent over this period, their alternative price index, only partially incorporating quality improvements, grew only 4.1 percent per year, falling relative to the total CPI at a rate of about 1.5 percent per year. Berndt, Bir, Busch, Frank and Normand (2000) estimate that the price of treating incidents of acute phase major depression declined in nominal terms by between 1.7 percent and 2.1 percent per year between 1991 and 1996, corresponding to a real rate of decline of more than 3 percent (though over a relatively short time period).

Table 1 solves the model for key results using this evidence and a range of values for $\theta$ to pin down $\mu$. More specifically, we consider values of $\theta$ of $0.20,0.25,0.30$, and 0.35 , and treatment price declines of 1 percent, 1.5 percent, and 2 percent. ${ }^{10}$ Table 1 then reports three statistics for each pair of parameter values. The first is simply the implied value for $\mu$. The second, $H / Y$ Factor, is the factor by which $H / Y$ increases in the model between 1960 and 1997 given this value for $\mu$. Using equation (14), $H / Y$ Factor is given by ${ }^{11}$

$$
\frac{H(97) / Y(97)}{H(60) / Y(60)} \approx \frac{e^{(\mu-g)(1997-1960)}}{a^{*}(97) / a^{*}(60)} .
$$

Finally, "Frac. Expl." reports that fraction of the actual increase in $H / Y$ explained by the model, equal to ("H/Y Factor" -1$) /(2.67-1)$, where

\footnotetext{
${ }^{10} \mathrm{An}$ important consideration in picking the range for the treatment price declines is that there is also a bias in the total CPI index. The papers considered above attempt to measure the bias in the treatment price index, but to the extent that the total CPI inflation rate is biased upwards, the real price declines implied by those studies may be overstated. This is why we limit the upper end of the range to two percent.

${ }^{11}$ We assume that the $\tau(t) \leq \bar{\tau}$ constraint is not binding so that $\ell^{*}=0$.
} 
TABLE 1.

Explaining the Rise in $H / Y$

\begin{tabular}{lllll}
\hline & \multicolumn{3}{c}{ Values of $\theta$} & \\
\cline { 3 - 4 } & 0.20 & 0.25 & 0.30 & 0.35 \\
\hline
\end{tabular}

1. Treatment price decline: $\mu-\theta / \alpha=-0.01$

$\begin{array}{ccccc}\mu & 0.0295 & 0.0393 & 0.0492 & 0.0591 \\ H / Y \text { Factor } & 1.38 & 1.98 & 2.86 & 4.12 \\ \text { Frac. Expl. } & 0.23 & 0.59 & 1.11 & 1.87\end{array}$

2. Treatment price decline: $\mu-\theta / \alpha=-0.015$

$\begin{array}{ccccc}\mu & 0.0245 & 0.0343 & 0.0442 & 0.0541 \\ H / Y \text { Factor } & 1.14 & 1.65 & 2.37 & 3.42 \\ \text { Frac. Expl. } & 0.09 & 0.39 & 0.82 & 1.45\end{array}$

3. Treatment price decline: $\mu-\theta / \alpha=-0.02$

$\begin{array}{ccccc}\mu & 0.0195 & 0.0293 & 0.0392 & 0.0491 \\ H / Y \text { Factor } & 0.95 & 1.37 & 1.97 & 2.84 \\ \text { Frac. Expl. } & -0.03 & 0.22 & 0.58 & 1.11\end{array}$

Note: " $H / Y$ Factor" is the factor by which $H / Y$ increases, calculated directly from equation (20). "Frac. Expl." denotes the fraction of the actual increase in $H / Y$ explained by the model and is equal to ("H/Y Factor" -1$) /(2.67-1)$, where 2.67 is the actual factor increase in the U.S. data between 1960 and 1997. 
$13.6 / 5.1=2.67$ is the actual factor increase in the U.S. data between 1960 and 1997.

The results in Table 1 suggest that it is quite plausible that the "march of science" accounts for a substantial amount of the increase in health expenditures as a share of GDP. Without more accurate estimates of $\theta$ and the rate at which treatment prices decline, it is difficult to be precise about the exact explanatory power of the model. Nevertheless, across a wide range of parameter values the fraction is substantial. The main exception seems to be if, simultaneously, $\theta$ is small and treatment prices decline very rapidly.

\section{SIMULATING THE MODEL, PART II}

The results from the previous section suggest that advances in medical technologies can account for a substantial fraction of the increase in the aggregate health expenditure share. In this section, we pick specific parameter values for $\mu$ and $\theta$ and simulate the model over time to examine its predictions along other dimensions. In particular, we choose $\mu=0.0473$ and $\theta=0.2877$. The choice of $\mu$ means that the model will match exactly the increase in the U.S. health expenditure share. The choice of $\theta$ means that one quarter of health expenditures go for people in the last year of life. For completeness, the implied value of $\gamma$ given these choices is -0.0094 , so that quality-adjusted treatment prices decline at a rate of about 1 percent per year. As should be clear from the previous discussion, there is no reason to think these parameter values are exactly correct, and one should not read too much into the exact quantitative predictions they imply.

A couple of other parameters need to be calibrated in order to simulate the full model. The parameter $\sigma$ captures in a reduced-form fashion the individual's budget constraint limit on health expenditures. We assume, arbitrarily, that $\sigma=1 / 2$ so that individuals can spend no more than one 
FIGURE 4. Simulation Results for $\bar{\tau}=.10$

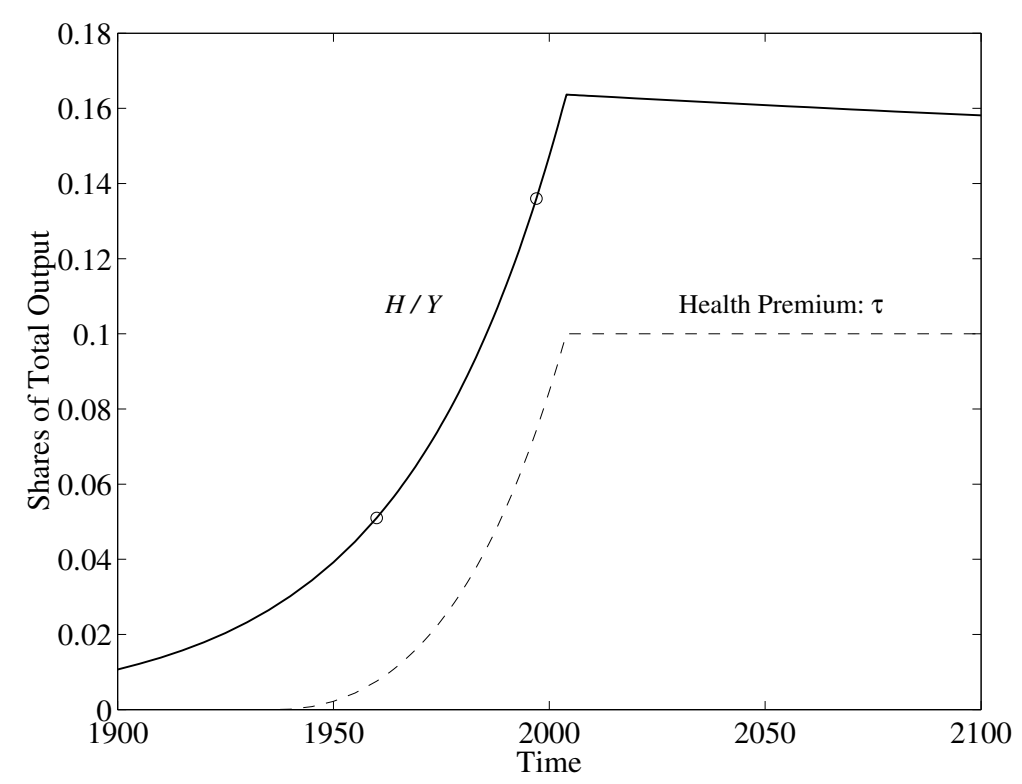

Note: The two circles in the figure indicate data points for the United States: an expenditure share of 5.1 percent in 1960 and 13.6 percent in 1997, when life expectancy was 66.6 and 73.9 years, respectively. See notes to Table 2.

half of per capita income on health. Many of the results below do not involve this parameter at all. The exception is in determining the level of transfer payments, where the sensitivity is direct and obvious: for any given aggregate level of spending, a higher value of $\sigma$ will reduce the amount of transfers required to support that level of spending.

Finally, the parameter value for $h_{0} / y_{0}$ has been chosen so that the simulation results match exactly U.S. aggregate health expenditures as a share of GDP in 1960. This leads to a value of $h_{0} / y_{0}=.9771$.

Figure 4 shows the aggregate health expenditure share and the transfer rate $\tau$ over time for a simulated economy. Statistics on $H / Y$, life expectancy, and other variables are reported in Table 2. 
TABLE 2.

Simulation Results

\begin{tabular}{|c|c|c|c|c|c|c|c|}
\hline Year & $H / Y$ & $\tau$ & $\bar{a}$ & $a^{*}$ & $\ell^{*}$ & $\begin{array}{c}\text { Fraction } \\
\text { Subsidized }\end{array}$ & $h\left(\ell^{*}\right) / y$ \\
\hline \multicolumn{8}{|c|}{$\bar{\tau}=.10$} \\
\hline 1900 & 0.011 & 0.000 & 54.8 & 54.8 & 0.000 & 0.000 & 0.168 \\
\hline 1920 & 0.018 & 0.000 & 58.7 & 58.7 & 0.000 & 0.000 & 0.302 \\
\hline 1940 & 0.030 & 0.000 & 62.7 & 62.7 & 0.000 & 0.005 & 0.544 \\
\hline 1960 & 0.051 & 0.008 & 66.6 & 66.6 & 0.000 & 0.035 & 0.977 \\
\hline 1970 & 0.066 & 0.017 & 68.6 & 68.6 & 0.000 & 0.050 & 1.310 \\
\hline 1980 & 0.087 & 0.032 & 70.5 & 70.5 & 0.000 & 0.064 & 1.756 \\
\hline 1990 & 0.113 & 0.054 & 72.5 & 72.5 & 0.000 & 0.077 & 2.355 \\
\hline 1997 & 0.136 & 0.074 & 73.9 & 73.9 & 0.000 & 0.086 & 2.891 \\
\hline 2000 & 0.147 & 0.085 & 74.5 & 74.5 & 0.000 & 0.090 & 3.157 \\
\hline 2010 & 0.163 & 0.100 & 76.5 & 75.9 & 0.598 & 0.094 & 3.564 \\
\hline 2050 & 0.161 & 0.100 & 84.4 & 79.8 & 4.551 & 0.087 & 3.693 \\
\hline 2100 & 0.158 & 0.100 & 94.2 & 84.7 & 9.499 & 0.079 & 3.854 \\
\hline \multicolumn{8}{|c|}{$\bar{\tau}=.05$} \\
\hline 1900 & 0.011 & 0.000 & 54.8 & 54.8 & 0.000 & 0.000 & 0.168 \\
\hline 1920 & 0.018 & 0.000 & 58.7 & 58.7 & 0.000 & 0.000 & 0.302 \\
\hline 1940 & 0.030 & 0.000 & 62.7 & 62.7 & 0.000 & 0.005 & 0.544 \\
\hline 1960 & 0.051 & 0.008 & 66.6 & 66.6 & 0.000 & 0.035 & 0.977 \\
\hline 1970 & 0.066 & 0.017 & 68.6 & 68.6 & 0.000 & 0.050 & 1.310 \\
\hline 1980 & 0.087 & 0.032 & 70.5 & 70.5 & 0.000 & 0.064 & 1.756 \\
\hline 1990 & 0.108 & 0.050 & 72.5 & 72.4 & 0.149 & 0.075 & 2.256 \\
\hline 1997 & 0.108 & 0.050 & 73.9 & 73.1 & 0.843 & 0.074 & 2.269 \\
\hline 2000 & 0.108 & 0.050 & 74.5 & 73.4 & 1.141 & 0.073 & 2.274 \\
\hline 2010 & 0.107 & 0.050 & 76.5 & 74.3 & 2.133 & 0.072 & 2.292 \\
\hline 2050 & 0.105 & 0.050 & 84.4 & 78.3 & 6.104 & 0.066 & 2.363 \\
\hline 2100 & 0.102 & 0.050 & 94.2 & 83.1 & 11.073 & 0.061 & 2.450 \\
\hline
\end{tabular}

Note: Simulation results with the following parameters: $\alpha=5.0685, \mu=.0473, \sigma=1 / 2$, $g=.018, y_{0}=1, h_{0} / y_{0}=.9771$. "Fraction Subsidized" denotes the fraction of the population receiving a health expenditure subsidy, $\left(\bar{\ell}-\ell^{*}\right) / a^{*}$. 
Several results from the simulation deserve mention. First, the aggregate health expenditure share grows rapidly until $\tau(t)=\bar{\tau}$, here set (arbitrarily) to 10 percent. As long as $\ell^{*}=0$, that is as long as all technologies are utilized, the exponentially rising cost of the frontier technology causes the aggregate health share to increase. Mathematically, this result is already familiar from equation (14): the health expenditure share of decedents grows at rate $\mu-g$ while life expectancy grows only linearly. Second, there exist parameter values such that the simulation matches exactly the level of $H / Y$ in 1960 and 1997, and the rate of increase in between.

Third, $\bar{\tau}$ reflects the maximum level of resources that this economy is willing to transfer from healthy people to sick people. In practice, $\bar{\tau}$ is the outcome of a very complicated political economy problem, and it could change over time as the political calculus changes. However, following our basic approach in this model, we treat this political outcome parametrically. What one sees from the simulation is that once this political constraint is binding, the dynamics of $H / Y$ change drastically. Before the constraint binds, the aggregate health expenditure share grows rapidly as society transfers an increasing fraction of resources from the healthy to the sick. Once this source of additional funding is exhausted - which in this simulation happens in the year 2004 - the rise in $H / Y$ comes to a halt and in fact the aggregate health share begins a slow, steady decline. The source of this decline was apparent back in equation (14): if growth in the expenditure share at the end of life is halted (or at least slowed considerably), the dilution effect takes over. Because of technological progress, the rise in life expectancy means that the population consists of an increasing fraction of healthy people. In the simulation in Figure 4, this dilution effect dominates after the $\bar{\tau}$ constraint is hit. $H / Y$ declines, asymptoting to the value given by $\bar{\tau}$ itself, since by construction society is always spending at least this much of its income on health. 
But if the individuals near death are always spending $\sigma(1-\tau) y$ of their own resources on health, why does the aggregate share asymptote to $\bar{\tau}$ ? The answer is suggested by the "Fraction Subsidized" column in Table 2. In particular, notice that the fraction of the population benefitting from the subsidy first rises and then declines after the $\bar{\tau}$ constraint becomes binding. That is, the full amount of the subsidy becomes increasingly concentrated within the population after the constraint is met, so that health expenditures financed by the resources of these people shrinks to zero. A political economy implication of this result is that it may be difficult to sustain a positive value of $\bar{\tau}$ in the long run.

The policy parameter $\bar{\tau}$ obviously plays a key role in the simulation results. If it were set higher, the rapid growth of $H / Y$ would continue longer. And if it were set lower, $H / Y$ would peak sooner.

There is a sense in which the "multiplier" associated with a change in $\bar{\tau}$ is large. Figure 5 examines the consequences of cutting $\bar{\tau}$ in half, from .10 to .05 , starting from the beginning of the simulation. In this case, the constraint that $\tau(t)<\bar{\tau}$ starts to bind in 1989 rather than in 2004, and the aggregate health expenditure share peaks in that year at 10.8 percent, or 5.8 percent above $\bar{\tau}$. Notice that this is less than the gap when $\bar{\tau}=.10$, which equals $16.3-10=6.3$ percent: the transfer rate is associated with a multiplier that is greater than one. The intuition for this result is that transfer payments increase the number of people near death, and these people themselves spend some of their own resources on health. As one would expect, a higher value of $\sigma$ will increase this multiplier.

The substantial reduction in health expenditures as a share of GDP associated with the lower transfer rate of $\bar{\tau}=.05$ has a relatively small effect on life expectancy, as can be seen in the lower panel of Table 2. In the year 1997, for example, life expectancy in the original simulation matched U.S. life expectancy at 73.9 years. In the simulation with $\bar{\tau}=.05$, life ex- 
FIGURE 5. Simulation Results for $\bar{\tau}=.05$

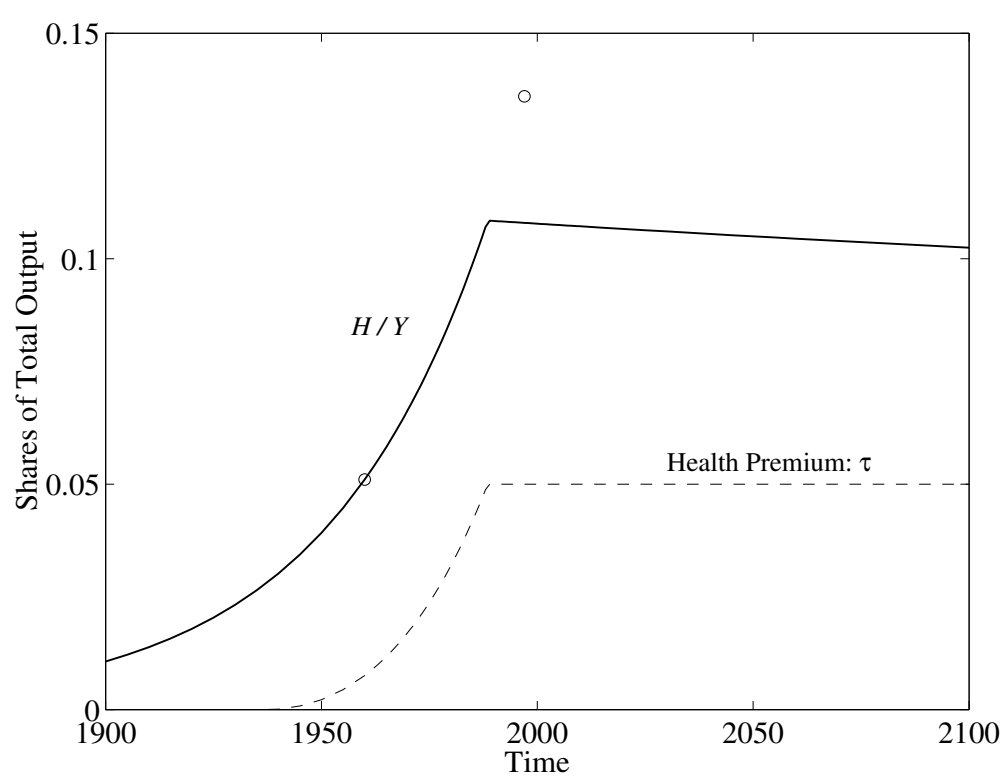

Note: See notes to Figure 4.

pectancy in 1997 is just under one year less at 73.1 years. About 2.8 percent of GDP goes to pay the health expenses of people living the additional 0.8 years, suggesting a ratio of $2.8 / 0.8=3.5$ percent of GDP for each additional year of life expectancy.

This number can be calculated analytically in the following way. Adding one year of life expectancy to the population raises total health expenditures by

$$
\int_{\ell^{*}-1}^{\ell^{*}} h(\ell, t) d \ell=\frac{1}{\theta} h\left(\ell^{*}, t\right)\left(e^{\theta}-1\right) .
$$

Dividing this by total GDP, one sees that the additional cost is approximately $H / Y \times\left(e^{\theta}-1\right)$. With $\theta=.2877$ and $H / Y=10.8$, we get 3.6 percent of GDP. 
Alternatively, if we start with the observed $H / Y=.136$ in 1997 , this suggests a cost of $.136 \times e^{.2877-1}=.045$, or 4.5 percent of GDP for each additional year of life expectancy at the margin in 1997. Is this a lot or a little? Obviously the answer depends on preferences and other complicated considerations. However, we can shed light on this question in two ways. In 1997, per capita GDP was about $\$ 28,000$, and life expectancy was $a^{*}(1997)=73.9$ years. Multiplying these two numbers together yields $Y(t)=a^{*}(t) y(t)$ of about 2 million dollars, and 4.5 percent of this is $\$ 93,000$. This number is right in the middle of economists' estimates of the value of one year of life, which range from about $\$ 50,000$ to about $\$ 150,000$ according to Cutler and McClellan (2001).

The range of uncertainty surrounding such a calculation is large. Still the calculation is useful in that it suggests that spending 13.6 percent of GDP on health care is not obviously crazy. This result is consistent with related findings in the literature. Cutler and McClellan (2001) estimate that between 1950 and 1990, the present discounted value of the amount an individual could expect to spend on medical care over her entire life rose by $\$ 35,000$. During the same period, life expectancy increased by about seven years, which Cutler and McClellan claim is worth a present value of $\$ 130,000$. They conclude that if health expenditures explain more than about a quarter of the rise in life expectancy, then these benefits from increased health spending exceeded the costs. ${ }^{12}$

A recent report by a panel of experts on the technical merits of the Medicare Trustees' financial projections provides some startling forecasts related to health expenditures as a share of GDP. ${ }^{13}$ For example, the middle-range estimate - which assumes health expenditures grow at a rate 1 percent

\footnotetext{
${ }^{12}$ Other related calculations on the gains from the increase in life expectancy can be found in Murphy and Topel (2002) and Nordhaus (2002).

${ }^{13}$ See Technical Review Panel on the Medicare Trustees Reports (2000).
} 
faster than GDP - forecasts an aggregate expenditure share of $25 \%$ in 2050 and $38 \%$ in 2075, and the report states that "The Panel does not view this [latter] figure as implausible" (p. 39).

The model sheds light on this forecast in two ways. First, the model is potentially consistent with this forecast as long as society is willing to continually transfer more and more resources to people near the end of life, i.e. as long as $\bar{\tau}$ is sufficiently large. On the other hand, the model suggests a reason to be cautious about these kind of forecasts: the dynamics of $H / Y$ look very different before and after the $\bar{\tau}$ constraint becomes binding. If society decides to cap the transfer rate, these forecasts could be far from the mark.

Second, the calculations on the value of a year of life implicitly provide an upper bound on the fraction of GDP that might optimally be spent on medical care. For example, in the first calculation the value of $\$ 93,000$ was right in the middle of the typical estimates of the value of a year of life, perhaps indicating that the upper bound on $H / Y$ is not too far away.

\section{ADDITIONAL EVIDENCE}

We now turn to other pieces of evidence that can be used to assess the success of the model, the first of which involves health-related transfer payments. The aggregate health expenditure share starts to decline when the economy hits the constraint on transfers, $\bar{\tau}$. Interestingly, U.S. federal and state health expenditures as a share of GDP show a pattern not unlike that of $\tau(t)$ in the simulation. In 1960, this share was 1.3 percent. It rose steadily over time before peaking in 1995 at 6.3 percent and declining slightly to 6.1 percent in $1998 .{ }^{14}$ As shown in Figure 1, this peak in the public health share corresponds closely to the peak in $H / Y$, as predicted by

\footnotetext{
${ }^{14}$ These numbers are computed from National Center for Health Statistics (2000), Table 115 , page 322 .
} 
FIGURE 6. Changes in Health Share vs. Changes in Life Expectancy

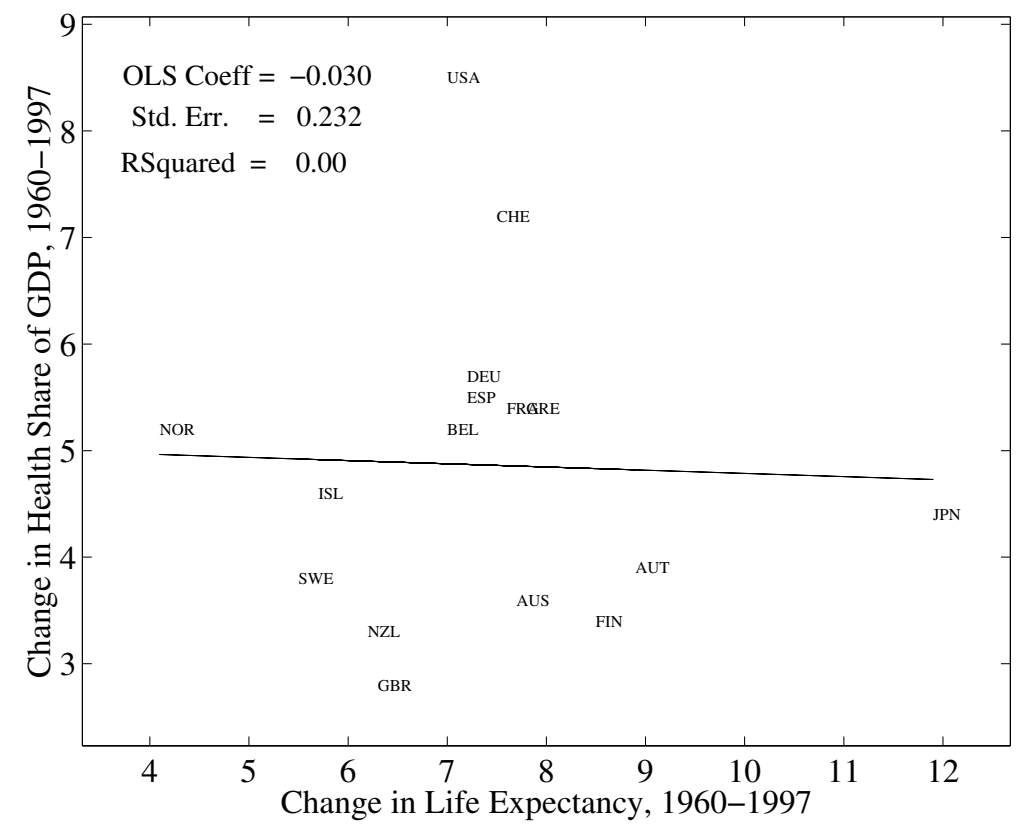

Notes: Data from OECD (2000), Tables 7 and 16. Life Expectancy is for males only.

the model. Changes in U.S. Medicare policy and the increased emphasis on managed care during the 1990s might be thought of as factors that affected $\bar{\tau}$.

One piece of evidence that is commonly thought to be a problem for a model like this is that there is essentially no correlation between the changes in life expectancy and the changes in the health expenditure share throughout the OECD. The levels are strongly correlated, as shown earlier in Figure 2, but the changes are not, as documented in Figure 6. Countries in which life expectancy increased by a large amount did not on average experience large increases in health expenditures as a share of GDP. 
In fact, careful reflection suggests that the model may be consistent with this evidence. It is important to appreciate that the model does not predict a monotonic relationship between changes in life expectancy and changes in $H / Y$. Recall from Figure 4 that $H / Y$ can either rise or fall as life expectancy increases, depending on whether or not the $\tau(t) \leq \bar{\tau}$ constraint is binding. An economy that is unconstrained by $\bar{\tau}$ will see increases in the health share associated with technologically-driven gains in life expectancy but an economy that has reached its $\bar{\tau}$ would experience decreases in the health share associated with such gains. Consider the following comparison. The United States and the United Kingdom had similar changes in life expectancy between 1960 and 1997, which rose by about 7 years in both countries. In contrast, the increases in $H / Y$ were vastly different: $H / Y$ rose by more 8 percentage points in the United States but only by about 3 percentage points in the U.K. A possible explanation of this marked difference is that the U.S. let technological considerations determine its transfer payments while the U.K. limited increases in $\tau(t)$. As discussed earlier, large differences in aggregate health expenditures are typically associated with small differences in life expectancy in the model. These differences are small enough that they could easily be swamped by other considerations such as demography and income distribution. ${ }^{15}$

\section{CONCLUSION}

Why have health expenditures as a share of GDP been rising in the United States and throughout the OECD? This paper considers an explanation in

\footnotetext{
${ }^{15}$ More generally, differences in public health, nutrition, diet, and economic growth may help to explain some of the differences in life expectancy gains across countries. For example, both Japan and Norway had similar changes in health expenditure shares, but remarkably different changes in life expectancy. This could be related to the fact that over the period 1960 to 1992, Japan was one of the world's fastest growing countries, and to the nutritional gains that were associated with this growth.
} 
which health expenditures and life expectancy are endogenous variables driven by technological progress. Advances in medicine permit people to spend resources on health care in order to extend life. Starting from initial conditions in which very little is spent on health care because of the absence of such opportunities, this naturally leads to an increase in the health expenditure share. Moreover, advances in life expectancy permit people to live to face more serious medical problems, such as heart attacks and hip replacements, that in turn are eventually cured, but only at a price.

Combining this theory of the effects of technological change with a transfer program that allows health expenditures at the end of life to rise to four times per capita income produces a framework that is broadly consistent with the following stylized facts:

1. Medicare expenditures rise sharply as life expectancy declines in a cross section, at a rate of about 10 percent until life expectancy falls to 3 years and then at a rate of nearly 45 percent in the last couple of years of life.

2. The fraction of Medicare expenditures accounted for by people in the last year of life is about 30 percent, and this number is surprisingly stable over time.

3. While as much as 4 percent of U.S. GDP may go to pay the health expenses of people in the last year of life, it is not at all clear that this reflects an inefficiency in the health care system.

4. Technological progress reduces the quality-adjusted cost of treating specific medical conditions, at a rate that seems to be about one or two percent per year.

5. Life expectancy in the United States since 1960 has risen at a rate of about 2 years every decade. 
6. Health expenditures as a share of GDP have risen from 5.1 percent in 1960 to 13.6 percent in 1997 . At least half, and most likely three-quarters or more of this change seems to be driven by the "march of science" and medical advances.

7. This trend is present throughout the OECD countries, where the typical increase in health expenditures is from 4 percent of GDP to about 8 percent.

8. Health-related transfer payments as a share of GDP have increased substantially.

9. Across countries, there is very little correlation between changes in life expectancy and changes in health expenditures as a share of GDP.

Simulations of the model suggest that a critical determinant of health expenditures as a share of GDP is the willingness of society to transfer resources to people near the end of life. As long as this willingness is unconstrained, the health share in the model rises rapidly. On the other hand, once a cap on these transfers is reached, the model predicts a halt to the rising health share and even a slow, gradual decline to a long-run level corresponding to the transfer rate. The source of this decline is a "dilution effect" associated with rising life expectancy. There are always five years worth of people with life expectancies of five years or less. As life expectancy rises, the fraction of the population accounted for by these people declines. If the expenditure share of these high-cost people is kept from growing too rapidly, the increasing fraction of low-cost healthy people reduces the aggregate expenditure share.

The model clearly has a number of serious limitations. Most obviously, it treats in a reduced-form fashion a number of key parameters, including $\alpha, \mu, \theta, \sigma$, and $\bar{\tau}$. A productive avenue for future research is to examine the microfoundations that determine these parameter values. Such a study would be forced to confront the important but complicated effects 
of health insurance, the relation between life expectancy and technological progress, and the political economy of health-related transfer payments, not to mention the interactions between these phenomena. As just one interesting example, Weisbrod (1991) emphasizes the role played by the nature of health insurance in determining the rate and direction of technological progress.

\section{APPENDIX}

\section{Health expenditures in the last year of life}

This appendix calculates the fraction of total health expenditures that are associated with people in the last year of life. Let $H(a, b, t)$ denote health expenditures by people with life expectancies in the range $[a, b]$. Then,

$$
\begin{aligned}
H\left(\ell^{*}, \ell^{*}+1, t\right) & =\int_{\ell^{*}}^{\ell^{*}+1} h(\ell, t) d \ell \\
& =\frac{1}{\theta}\left(h\left(\ell^{*}, t\right)-h\left(\ell^{*}+1, t\right)\right) \\
& =\frac{1}{\theta} h\left(\ell^{*}, t\right)\left(1-e^{-\theta}\right) .
\end{aligned}
$$

From equation (14), this relation can be rewritten as

$$
\frac{H\left(\ell^{*}, \ell^{*}+1, t\right)}{Y(t)} \approx \frac{H(t)}{Y(t)} \times\left(1-e^{-\theta}\right) .
$$

Health expenditures in the last year of life as a share of GDP are proportional to $H / Y$ at rate $1-e^{-\theta} \approx \theta$. This factor, in turn, is then the fraction of total health expenditures associated with people in the last year of life.

\section{REFERENCES}

Baumol, William J., "Macroeconomics of Unbalanced Growth: The Anatomy of Urban Crisis," American Economic Review, June 1967, 57, 415-426.

Berndt, Ernst R., Anupa Bir, Susan H. Busch, Richard G. Frank, and Shaon-Lise T. Normand, “The Medical Treatment of Depression, 1991-1996: Productive 
Inefficiency, Expected Outcome Variations, and Price Indexes,” 2000. NBER Working Paper No. 7816.

Cutler, David M. and Mark McClellan, "Is Technological Change in Medicine Worth It?," July 2001. Harvard University mimeo.

__ , Mark B. McClellan, Joseph P. Newhouse, and Dahlia Remler, "Are Medical Prices Declining? Evidence from Heart Attack Treatments," Quarterly Journal of Economics, November 1998, 113 (4), 991-1024.

Lubitz, James D. and Gerald F. Riley, "Trends in Medicare Payments in the Last Year of Life," New England Journal of Medicine, April 1993, 328 (15), 10921096.

Lubitz, James, James Beebe, and Colin Baker, "Longevity and Medicare Expenditures," New England Journal of Medicine, April 1995, 332 (15), 999-1003.

Miller, Tim, "Increasing Longevity and Medicare Expenditures," Demography, May 2001, 38 (2), 215-226.

Murphy, Kevin M. and Robert Topel, "The Economic Value of Medical Research.” In Exceptional Returns (Murphy and Topel, eds 2002). Forthcoming.

__ and __ , eds, Exceptional Returns, University of Chicago Press, 2002. Forthcoming.

National Center for Health Statistics, Health, United States 20002000.

Newhouse, Joseph P., "Medical Care Costs: How Much Welfare Loss?,' Journal of Economic Perspectives, Summer 1992, 6 (3), 3-21.

Nordhaus, William D., "The Health of Nations: The Contribution of Improved Health to Living Standards.” In Murphy and Topel, eds (2002). Forthcoming.

OECD, "OECD Health Data 2000 - A Comparative Analysis of 29 Countries," http://www.oecd.org/els/health/software/fad.htm 2000. ISBN 92-64-06876-7.

Shapiro, Irving, Matthew D. Shapiro, and David W. Wilcox, "Measuring the Value of Cataract Surgery," 1999. University of Michigan mimeo.

Technical Review Panel on the Medicare Trustees Reports, "Review of Assumptions and Methods of the Medicare Trustees' Financial Projections," December 2000. Mimeo, downloaded from http://www.hcfa.gov/pubforms/actuary/TechnicalPanel/.

Triplett, Jack E., "What's Different about Health: Human Repair and Car Repair in National Accounts and in National Health Accounts," in Ernst Berndt and David Cutler, eds., Medical Care Output and Producitivity, University of Chicago Press, forthcoming. 
— and Barry P. Bosworth, "Productivity in the Services Sector," 2000. Brookings Institution mimeo.

Weisbrod, Burton A., "The Health Care Quadrilemma: An Essay on Technological Change, Insurance, Quality of Care, and Cost Containment," Journal of Economic Literature, June 1991, 29, 523-552.

White, Kevin M., "Longevity Advances in High-Income Countries, 1955-96," Population and Development Review, March 2002, 28 (1), 59-76. 